\title{
Loeiz Herrieu, de la langue des carnets à la langue littéraire
}

Loeiz Herrieu: from notebook language to literary language

\section{Anthony Heulin}

\section{Q OpenEdition}

\section{Journals}

Édition électronique

URL : https://journals.openedition.org//bl/1222

DOI : $10.4000 / \mathrm{lbl} .1222$

ISSN : 2727-9383

\section{Éditeur}

Université de Bretagne Occidentale - UBO

\section{Édition imprimée}

Date de publication : 1 juin 2015

Pagination : 343-361

ISBN : 979-10-92331-16-5

ISSN : 1270-2412

\section{Référence électronique}

Anthony Heulin, "Loeiz Herrieu, de la langue des carnets à la langue littéraire », La Bretagne

Linguistique [En ligne], 19| 2015, mis en ligne le 01 mai 2021, consulté le 22 mai 2021. URL : http:// journals.openedition.org//bl/1222 ; DOl : https://doi.org/10.4000/lbl.1222

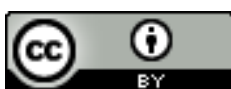

La Bretagne Linguistique est mise à disposition selon les termes de la Licence Creative Commons Attribution 4.0 International. 
Antony HeULIN*

\section{Loeiz Herrieu, de la langue des carnets à la langue littéraire}

\section{Présentation de Kammdro an Ankoù}

ne parenthèse. Le chercheur Daniel Carré, auteur de l'étude la plus complète ${ }^{1}$ à ce jour à propos de Loeiz Herrieu ${ }^{2}$, insiste sur le fait que celui-ci voulait considérer la guerre comme une parenthèse dans sa vie. Il s'avère que son récit Kammdro an Ankoù ${ }^{3}$, dont un extrait est l'objet de notre présentation, constitue également une parenthèse dans son œuvre littéraire. Ce récit, qui se présente sous la forme d'un journal de guerre, couvre la période du 29 juillet 1914 au 5 février 1919. Il est considéré comme étant le chef-d'œuvre de Loeiz Herrieu. En effet, le reste de sa production littéraire ne se distingue pas du tout-venant en langue bretonne publié à cette époque. Songeons par exemple à son recueil Dasson ur Galon ${ }^{4}$. Les poèmes qu'il contient

* Docteur en celtique, CRBC Rennes (EA 4451), Université Rennes 2/ueb.

1. Daniel CARRÉ, Loeiz Herrieu, un paysan breton dans la grande guerre. Analyse de sa correspondance avec son épouse, Thèse de Doctorat sous la direction de Yann-Ber Piriou, Université Rennes 2, 1999, non paginé.

2. 1879 (Caudan) - 1953 (Auray).

3. Loeiz Herrieu, Kammdro an Ankoù [Le carrefour de la mort], Brest, Al Liamm, 1994, $313 \mathrm{p}$.

4. Loeiz Herrieu, Dasson ur Galon [Les résonnances d'un cœur], Lorient, éditions Dihunamb, 1957, $253 \mathrm{p}$. 
sont un alignement d'images, de clichés qui trahissent une existence totalement corsetée par le souci des convenances.

Seuls l'expérience et le récit de la guerre posent Loeiz Herrieu en grand écrivain, auteur d'une unique œuvre marquante ${ }^{5}$. Car c'est paradoxalement dans cette terrible situation de privation de liberté d'homme qu'il trouve son espace de liberté littéraire. Grâce à la rédaction de carnets ${ }^{6}$ tout d'abord, dans lesquels il note chaque jour scrupuleusement ce qu'il lui arrive. Ces carnets vont être la source de Kammdro an Ankoù.

Notre présentation a pour objectif de mettre en lumière la nature et les causes du travail de réécriture de ces carnets, rédigés dans une langue spontanée, un premier jet destiné à être transformé en une œuvre écrite dans une langue littéraire.

Cette démarche est inédite chez Loeiz Herrieu. Fondateur et animateur de la revue Dihunamb, il a toujours eu à cœur de toucher un lectorat populaire en s'en tenant à une langue accessible au plus grand nombre ${ }^{7}$.

Les raisons qui poussent Loeiz Herrieu à faire de Kammdro an Ankoù une exception à ce principe d'écriture auquel il s'était toujours tenu jusque-là, tient à un ensemble de raisons que nous ne pouvons détailler ici. Précisons tout de même la principale d'entre elles : elle tient au fait que Loeiz Herrieu ne peut décrire la guerre à l'aide des images, des clichés auxquels il a recours habituellement dans

5. Caractéristique qu'il partage avec un autre auteur pacifiste, Erich-Maria Remarque qui ne produira aucune autre œuvre majeure après son célèbre $\dot{A}$ l'ouest rien de nouveau. Erich-Maria REMARQUE, À l'ouest rien de nouveau, traduit de l'allemand par Alzir Hella et Olivier Bournac, Paris, Stock, 2008, 254 p.

6. Loeiz Herrieu, Karnedoù brezel [Carnets de guerre], carnets de guerre originaux de Loeiz Herrieu, retranscrits par Daniel Carré, non paginés. 4 carnets de guerre originaux de Loeiz Herrieu datés du 23 mai 1915 au 4 février 1919, document inédit retranscrit par Daniel Carré. Merci à Daniel Carré de nous les avoir transmis. Par ailleurs, ces carnets ainsi que tous les documents d'archives relatifs à Loeiz Herrieu, sont désormais rassemblés au CRBC de Brest. Le travail de retranscription des carnets a été complété par un archivage complet du fonds effectué par Patrick Dréan.

7. Même si, comme le rappelle Daniel Carré dans sa thèse, le lectorat de Dihunamb, largement composé d'ecclésiastiques, touche en fin de compte peu le public populaire. 
ses écrits littéraires. La guerre est pour lui un contexte parfaitement inconnu. Elle le met donc face à un défi littéraire nouveau, parce qu'il n'a pas de mode d'emploi, de références en la matière, la Première Guerre mondiale, premier grand conflit moderne avec toutes les caractéristiques que cela sous-entend, utilisation de l'armement moderne, mobilisation d'hommes sans précédent, guerre de position, ne pouvant pas être décrite comme le furent les guerres précédentes.

Sur le plan littéraire, Kammdro an Ankoù s'inscrit dans la tradition du naturalisme français tel qu'Émile Zola le décrivait en évoquant Thérèse Raquin : «J'ai simplement fait sur deux corps vivants le travail analytique que les chirurgiens font sur les cadavres ${ }^{8}$. » Or, cette démarche de ne rien édulcorer est nouvelle dans la littérature en langue bretonne ${ }^{9}$. À cela s'ajoute le travail de réécriture des carnets de guerre pour faire de Kammdro an Ankoù une œuvre littéraire à part entière sans se soucier du lectorat ${ }^{10}$, ce qui rapproche Loeiz Herrieu des ambitions du mouvement «Gwalarn », dialectophobie en moins ${ }^{11}$.

8. Émile Zola, cité dans La Littérature française, Les grands mouvements littéraires $d u$ XIXe siècle, présentés par Carole Narteau et Irène Nouailhac, Paris, E. J. L-Librio, 2009, p. 63.

9. Nous ne pouvons détailler l'évolution vers la singularisation de l'expression littéraire en breton qui passe au XIX ${ }^{\mathrm{e}}$ siècle d'un mode collectif, anonyme, contes et chansons, à une production individuelle signée mais encore très stéréotypée avant de prendre la forme que nous lui connaissons depuis.

10. Le poète Yann-Ber Kalloc'h avait été le premier à montrer la voie dans ce sens avec ses poèmes écrits entre 1905 et 1917. Jean-Pierre CALLOC'H, À Genoux, Lais bretons, Paris, Éditions Plon-Nourrit, 1921, 234 p.

11. "Pour la première fois, une revue bretonnante fournira des travaux d'une irréprochable tenue littéraire, et, fermant la porte aux patois (même décorés du nom de dialectes), adoptera une langue de forme classique et une orthographe rigoureusement unique suivant l'exemple des Sketla Segobrani. " Extrait du Manifeste de Gwalarn, signé par Roparz Hemon et Olivier Mordrel, reproduit dans Francis FAVEREAU, Lennegezh ar brezhoneg en XXvet kantved, 1918-1944, levrenn 2, Breiz Atao hag ar re all el lennegezh (Anthologie de la littérature bretonne, 1918-1944, tome 2), Morlaix, Éditions Skol Vreizh, 2003, p. 239. Loeiz Herrieu, conserva toujours une distance avec les membres de Gwalarn, trop intransigeants à son goût. " On peut se demander, maintenant, qui l'emportera : de l'équipe du breton artificiel [Gwalarn], dont on ne peut nier le dynamisme ni l'activité ; mais qui a rompu, pour ainsi dire, avec la tradition et n'est plus en communion intime avec le peuple; ou de l'équipe du breton vivant, qui tient à ne pas creuser de fossé entre elle et le peuple breton, tout en évoluant vers une 


\section{Des carnets de guerre à Kammdro an Ankoù}

L'analyse détaillée du processus de transformation des Carnets de guerre de Loeiz Herrieu en œuvre littéraire, leur relation avec sa correspondance, le contexte de publication de Kammdro an Ankoù dans Dihunamb, mériteraient une étude d'envergure, sur le modèle du travail mené par toute une équipe de recherche sous la direction du professeur Helmut Kiesel ${ }^{12}$, à partir du journal de guerre d'Ernst Jünger ${ }^{13}$. Faute de temps et d'une équipe de chercheurs disposée à mener cette démarche avec la totalité de l'œuvre, nous nous contentons de comparer un extrait des Carnets avec Kammdro an Ankoù.

Afin de préciser le champ de notre analyse, il convient de définir ce qu'est la langue littéraire. Le théoricien de la littérature Jean Starobinski, dans sa présentation des études de style de Léo Spitzer, donne une définition de l'écrivain et de la littérature à laquelle nous nous référons :

« Notre goût exige que l'écrivain ait une voix à lui, et qu'il la pose d'une manière inimitable : allons jusqu'à dire que notre culture admet très communément l'idée de la littérature comme processus continuel de "déviance" : la littérature, c'est le collège discordant des voix et des écritures sans égales. L'écart est donc

littérature plus sélectionnée ? Dans l'intérêt de la littérature bretonne, souhaitons tout simplement que la première abandonne une partie de son intransigeance, pour rétablir le contact avec notre peuple, par des œuvres plus à sa portée, écrites dans une langue châtiée, mais correcte aussi et accessible à un plus grand nombre de lecteurs. Et que la seconde, tout en continuant à faire de la langue vivante, l'outil de sa pensée, abandonne cette fade littérature sans relief, sans sincérité, qui, trop longtemps, fut à la mode parmi les auteurs contemporains, les écrivains des deux équipes y gagneront une saine renommée et la reconnaissance des générations à venir. Notre littérature nationale y trouvera l'unité qui lui manquera et une force de rayonnement dont on ne peut supputer les heureuses conséquences. » Loeiz HerRIEU, La littérature bretonne des origines au XXe Siècle, Hennebont, Éditions de Dihunamb, 1943, p. 110-111.

12. Université de Heildelberg (Allemagne). Recherche menée grâce au soutien financier de la Frietz-Thyssen-Stiftung.

13. Ernst JÜNGER, Kriegstagbuch 1914-1918 (Journal de guerre 1914-1918), Helmut Kiesel (dir.), Stuttgart, Klett-Cotta, 2010, 654 p. et Ernst JÜNGER, Orages d'acier (In Stahlgewittern), traduit de l'allemand par Henri Plard, Paris, Christian Bourgois, 2013, 380 p. 
de règle, et seule reste imprévue la direction qu'il prendra chez un nouvel auteur ${ }^{14}$.»

À partir de cette définition, la langue littéraire est donc le matériau qui dit la littérature, par la voix de l'écrivain. Elle se distingue de la langue quotidienne, administrative, en ce qu'elle est à la fois une voix singulière qui s'exprime dans une langue singulière. Dans ses carnets, Loeiz Herrieu utilise une langue quotidienne, non retravaillée $^{15}$. Elle est une langue littéraire en devenir, un brouillon. Le travail qu'il opère à partir de ce matériau exprime son désir de se démarquer de sa forme initiale pour lui donner une nouvelle forme stylisée. C'est cette langue hors du commun que l'on peut nommer langue littéraire.

Ajoutons également que la langue littéraire présente souvent la particularité d'être destinée à être diffusée. Cette volonté de confrontation de l'auteur avec le lecteur provoque un phénomène d'interprétation de ses écrits par ce dernier. En somme, cette liberté d'interprétation répond à celle de l'auteur.

Nous avons choisi la date du 28 avril 1915 pour illustrer notre présentation. Le récit non retravaillé est contenu dans le premier carnet retrouvé, qui couvre la période du 23 avril 1915 au 27 avril 1916 ${ }^{16}$. Dans Kammdro an Ankoù, le 28 avril 1915 se trouve au chapitre V, intitulé Àr dalbenn-brezel Reims hag an Aisne [Sur le front de Reims et de l'Aisne], qui est un long chapitre ${ }^{17}$. Il couvre la période du 5 octobre 1914 au 15 juillet 1915. Pour information, notre présentation ne se situant pas directement dans le champ historique, cette période se situe juste après la bataille de la Marne qui a eu lieu en septembre 1914. Le rapport de la journée du 28 avril est

14. Jean Starobinski dans Leo SPITZER, Études de style, précédé de Leo Spitzer et la lecture stylistique par Jean Starobinksi, Paris, Gallimard, 1970, p. 23-24.

15. Ce qui ne l'empêche pas d'être rédigée car nous avons affaire à un homme qui écrit quotidiennement depuis une vingtaine d'années. Ce travail d'écriture constant explique la forme parfois très proche des carnets et du récit publié.

16. Loeiz Herrieu a vraisemblablement rédigé un ou plusieurs autres carnets à partir d'août 1914 jusqu'en avril 1915 mais ces carnets n'ont malheureusement pas été retrouvés. Soulignons et remercions encore le patient et précieux travail de retranscription tapuscrite des carnets retrouvés par Daniel Carré.

17. Loeiz Herrieu, Kammdro an Ankoù [Le carrefour de la mort], op. cit., p. 31-68. 
sensiblement de la même longueur dans le carnet que dans Kammdro an Ankoù.

Le 28 avril 1915, Loeiz Herrieu se rend à Fismes dans la Marne, sans préciser pour quelle raison, et note ceci dans son carnet :

«28. - Hiniù e han hoah de Fismes. Trémen e hramb dré Euilly hag en ur arriù get sukrdi Villers Haute Rive é choman soéhet é huélet marteloded koh étal er pont. Soéhetoh onn hoah a pe dostan dehè é huélet é ma labourizion porh int. Deit int a houdé miz devéhan er blé aveit seùel hag gobér a neùé er ponteu diskaret a pe oè er Germaned é tonet é Frans.

Bout e zo ag er hantér (?) anehè hag a gosté en Oriant. Un adjudant labourér porh e zo doh ou houmandein hag un ingénieur gusket èl ur Lieutenant de vaisseau.

E Fismes en anderù-men de 3 ér é interrér 15 sudard lahet deu zé kent é gar Fismes get ur bomb taulet d'un aerlestr hag e ziskoé bout un aerlestr a Frans. En ou mesk é oè sudarded ag er 88 hag ur serjant Durand a neùéso hanùet ér ravitaillemant léh ma um gavé a drès er peurkèh. Donet e hra hoah un aerlestr germanek de valé drest ker meit monet e hra unan ag hor ré ar é lerh ha ne déhas ket turel bomb erbet.

Guélet e hran deu di e zo koéhet bombeu arnehé. Goal auzet int, muioh memb eit get obuseu.

E tonet d'er gér é kavan hoah er labourizion porh é kampen un tachad aveit lakat ou hamakeu a skrebill aveit monet de gousket. (*) Chom e hreint aman un tér suhun benak.

E Euilly é ma arriù er 144 de gemér hor lèh. Lan é er vorh anehé. Kement-men e senefi, kredan, ne vemb ket galùet d'hobér labour en active. Arriù zo genemb territ. ag en 218 e zo lammet ag en active aveit lakaat er 88 er lod muian anehè e zo a gosté Pau. Tud didrous, um gav un tammig divroet émesk hor Bretoned e zalh de gonz ieh er vro. »

«28. - Aujourd'hui je vais encore à Fismes. Nous passons par CEuilly et tout en arrivant à la sucrerie de Villiers Haute Rive je reste étonné en voyant des vieux marins près du pont. Je suis encore plus étonné lorsque je m'approche d'eux en voyant que ce sont des ouvriers de l'arsenal. Il sont venus depuis le dernier mois de l'année pour construire et rénover les ponts détruits lorsque les Allemands étaient en train de venir en France. 
Il y en a la moitié (?) d'entre eux du côté de Lorient. Un adjudant ouvrier de l'arsenal est à leur commandement et un ingénieur $(f)^{18}$ vêtu comme un Lieutenant de vaisseau $(f)$.

À Fismes cet après-midi à 3 heures on enterre 15 soldats tués deux jours avant à la gare de Fismes par une bombe lancée par un avion qui ressemblait à un avion de France. Parmi eux il y avait des soldats du $88^{\mathrm{e}}$ et un sergent Durand nouvellement nommé au ravitaillement où il se trouvait bien le pauvre. Il vient encore un avion allemand se promener au-dessus de la ville mais un des nôtres le prend en chasse et il ne parvient à lancer aucune bombe.

Je vois deux maisons sur lesquelles sont tombées des bombes. Elles sont en mauvais état, plus même que par des obus.

En venant à la ville je trouve encore des ouvriers de l'arsenal en train d'arranger un terrain pour suspendre leur hamac pour aller dormir (*). Ils resteront ici quelque trois semaines.

À Euilly le 144 est arrivé pour prendre notre place. Le bourg est plein d'eux. Ceci signifie, je crois, que nous ne serons pas appelés à faire de travail en active (f). Il nous arrive des territ. (f) du 218 qui sont enlevés de l'active (f) pour mettre au 88 : la plupart d'entre eux est du côté de Pau. Des hommes calmes, qui se trouvent un peu dépaysés parmi nos Bretons qui continuent de parler la langue du pays ${ }^{19}$. »

Voici maintenant la version retravaillée, publiée, de Kammdro an Ankoù :

«28-05-1915 - É vonet da Fismes hiziv eh on boemet-mat é welet un nebeud martoloded kozh é labourat e-tal pont Villers. Labourizion-porzh int, daet du-mañ da adsevel ar pontoù. Anavout a ran lod anezhe: a Lanester int. Emaint dre-mañ a-c'houde c'hwec'h miz bennak.

E Fismes e tegouezhan just evit gwelet interamant 15 soudard lazhet er gar get ur vombezenn taolet ag un aerlestr hag a oa hañval-mat doc'h hor re. Unan bennak skrapet get ar Jermaned moarvat.

18. (f) : en français dans le texte.

19. Loeiz Herrieu, Karnedoù brezel [Carnets de guerre], retranscrits par Daniel Carré. Sauf indication contraire, toutes les traductions du breton en français sont de notre fait. Ces traductions prétendent simplement illustrer notre analyse du texte original sans autre ambition, la traduction de Kammdro an Ankoù représentant une entreprise extrêmement complexe. 
E-mesk ar geizh-se eh oa tud ag an 88vet. Unan anezhe, ur serjant, kaset du-mañ an deizioù kent hag eürus bout pellaet doc'h an talva, a zo daet amañ da glask e varv.

A pa zistroan da Beaurieux eh eus labour arall doc'h man gortoz : soudarded lamet a-douesk ar re yaouank en arbenn d'o oad a zo kaset deomp. Euskariz int, lod anezhe; tud didrouz, o-unan un tammig, hag a vourr é komz o yezh.

Kement-mañ a ra koñfort deomp : revendailh ne vezer ket soñjet d'ober genomp labour ar re yaouank. »

«28-05-1915 - En allant à Fismes aujourd'hui je suis bien surpris en voyant quelques vieux marins en train de travailler près du pont de Villers. Ce sont des ouvriers de l'arsenal, venus ici pour reconstruire les ponts. J'en connais quelques-uns : ils sont de Lanester. Ils sont par ici depuis quelque six mois.

Nous arrivons à Fismes juste pour voir l'enterrement de 15 soldats tués dans la gare par une bombe lancée d'un avion qui était semblable aux nôtres. Sûrement un volé par les Allemands.

Parmi ces pauvres-là il y avait des hommes du 88e. L'un d'entre eux, un sergent, envoyé ici quelques jours auparavant, qui était heureux de s'être éloigné du front, est venu ici chercher sa mort.

Lorsque je retourne à Beaurieux il y a un autre travail à m'attendre : des soldats retirés d'entre les jeunes à cause de leur âge nous sont envoyés. Ce sont des Basques, pour certains ; des hommes calmes, un peu seuls, qui aiment parler leur langue.

Ceci nous réconforte : à ce qu'il semble on n'a pas songé à nous faire faire le travail des jeunes ${ }^{20}$. »

\section{Analyse du récit brut des carnets}

Les contraintes de temps subies par Loeiz Herrieu sur le front où il est fourrier dans des conditions de vie extrême, sont immédiatement observables dans le récit du carnet de cette journée du 28 avril 1915. Alors qu'en 1932 ou 1933, à l'heure où il retravaille ce texte pour l'intégrer à Kammdro an Ankoù, bien qu'il soit encore une homme très occupé par la charge de Dihunamb et son engagement dans la défense du breton, il rédige ses nouvelles lignes dans la quiétude de

20. Loeiz Herrieu, Kammdro an Ankoù [Le carrefour de la mort], op. cit., p. 66. 
son bureau, l'esprit d'autant plus tranquille qu'un de ses fils vient de reprendre l'exploitation de sa ferme.

Quand Loeiz Herrieu consigne ses impressions le 28 avril 1915 au soir, il est certainement dans un état de fatigue avancée. Pendant cette journée, il a effectué un aller-retour entre Beaurieux et Fismes, en charrette. Cela correspond à une distance globale d'environ ${ }^{21}$ 28 kilomètres. En arrivant à Fismes, il assiste à un enterrement. Enfin, de retour à Beaurieux, son travail de fourrier l'oblige à traiter l'intégration de nouveaux arrivants dans son régiment. C'est seulement après ce travail qu'il rédige son journal comme il le fait et va le faire quotidiennement depuis son premier jusqu'au dernier jour de mobilisation. Ce qui donne une idée de la force de caractère et de la capacité d'auto-discipline de l'homme.

Nous ne nous étonnons donc pas de trouver un résumé brut de la journée où seuls des points finaux séparent des phrases ponctuées à la va-vite ${ }^{22}$.

"Soéhetoh onn hoah a pe dostan dehè é huélet é ma labourizion porh int », se traduit littéralement par "Je suis plus étonné encore lorsque j'approche d'eux en voyant que c'est/ qu'il y a des ouvriers de l'arsenal ils sont ». Remarquons également l'emploi de é $m a^{23}$, forme de situation au présent du verbe bout [être] normalement employé devant un sujet défini.

Néanmoins, cette syntaxe n'est pas fautive en breton ${ }^{24}$. Elle constitue ce que les linguistes nomment une structure clivée, dont l'objectif est de mettre en relief l'information exprimée ${ }^{25}$. Ainsi,

21. Nous soulignons environ n'ayant pas pu établir le distance précise en 1915, mais sachant que les conditions de circulation étaient de toute façon plus compliquées à cause du conflit. Source de calcul de l'itinéraire Beaurieux-Fismes via CEuilly : http://www.viamichelin.fr (consulté le 20 octobre 2013).

22. Nous avons traduit le passage original en rétablissant une syntaxe également peu académique mais en rendant le texte aussi compréhensible dans sa version française que bretonne.

23. Orthographié ema ou emañ en breton contemporain.

24. Nous nous devons ici de remercier les chercheurs Mélanie Jouitteau, Erwan Le Pipec et Francis Favereau qui nous ont apporté ces précisions lors de la communication de cette étude à l'UBO (séminaire La Bretagne Linguistique) le 13 décembre 2013.

25. Sur les structures clivées, nous renvoyons au site Internet ARBRES, animé par la linguiste Mélanie Jouitteau (https://arbres.iker.cnrs.fr/index. 
cette forme spécifiquement bretonne nous éclaire sur le cheminement de la pensée de Loeiz Herrieu. Il désire d'abord situer géographiquement ces ouvriers tout en les présentant comme tels : « Il y a des ouvriers de l'arsenal et eux, ce sont des ouvriers de l'arsenal. » Pourquoi dès lors, la modifie-t-il dans sa version définitive ? C'est vraisemblablement par une volonté d'hypercorrection que Loeiz Herrieu supprime cette version « trop » syntaxiquement bretonne par quelque chose qui se rapproche plus du français ${ }^{26}$.

Plus généralement, la syntaxe comme le vocabulaire, apparaissent parfois répétitifs et peu recherchés « (...)é choman soéhet é huélet marteloded koh étal er pont. Soéhetoh onn hoah a pe dostan dehè é huélet é ma labourizion porh int»; «(...) je reste étonné en voyant des vieux marins près du pont. Je suis encore plus étonné lorsque je m'approche d'eux en voyant que ce sont des ouvriers de l'arsenal ».

Des notes écrites dans l'instant peuvent être également sources de confusion. Ainsi, dans la dernière phrase : «Tud didrous, um gav un tammig divroet émesk hor Bretoned e zalh de gonz ieh er vro. " ; «Des hommes calmes, qui se trouvent un peu dépaysés parmi nos Bretons qui continuent de parler la langue du pays ».

Qui, des hommes calmes ${ }^{27}$ ou des Bretons, continuent à parler la langue du pays?

Ces observations confirment que Loeiz Herrieu rédige ces lignes dans l'urgence, sans peut-être même y réfléchir. Il fixe. L'important n'est pas encore de procéder à un travail sur la forme mais de poser ces notes comme autant d'aide-mémoires qui amorceront la résurgence de souvenirs plus détaillés lorsqu'il s'attellera à la rédaction de Kammdro an Ankoù. Nous pouvons comparer cette langue à celle de l'enfant qui a vu et vécu tant de choses extraordinaires pendant sa journée, que ses mots trébuchent dans sa bouche au moment il la

php?title=Structures_cliv\%C3\%A9es) (consulté le 16 décembre 2013). Le linguiste Erwan Le Pipec précise que ces structures peuvent également être désignées sous le terme de représentations anaphoriques du verbe.

26. Nous reprenons ici l'interprétation du phénomène par Jo Rio, confirmé par Francis Favereau et Daniel Carré.

27. Didrouz pourrait aussi être traduit par « silencieux », mais il ne nous semblait pas logique d'évoquer des hommes silencieux en train de... parler. 
raconte « à chaud », parce qu'il voudrait tout dire et ne rien oublier dans son récit, ne rien perdre. C'est un récit sans recul ni distance. Loeiz Herrieu connaît les exigences de l'écriture littéraire. Il sait que les premières notes sont semblables à ces carnets de croquis fébrilement remplis par les peintres, en attendant d'y voir apparaître le détail, la forme qui inspirera et guidera l'exécution du tableau tout entier. Dès lors, telle une esquisse, cette langue peut déraper, s'aventurer ici ou là sans remords puisqu'elle n'est en aucun cas destinée au public ${ }^{28}$. La première impression ou image qui revient à l'esprit, le premier mot qui passe pour la désigner est consigné. De ce fait, quand Loeiz Herrieu, contraint de rédiger à longueur de journée des rapports en français pour l'armée, ne trouve pas d'emblée le mot breton parce que ce mot ne recouvrait aucune réalité connue en Bretagne, il l'écrit dans la langue où il est le plus fréquemment entendu, c'est-à-dire en français, ainsi " en active », " territ. ", " ingénieur » ou « lieutenant de vaisseau », alors qu'il nomme sans problème les «labourizion porh ; ouvriers de l'arsenal », familiers à Lorient ${ }^{29}$.

Analyse de la transformation du récit brut des carnets en récit littéraire

Comment ces phrases écrites dans l'urgence dans une langue quotidienne, non recherchée vont-elles se transformer pour donner naissance à une langue littéraire travaillée et retravaillée dans le calme d'un bureau près d'une vingtaine d'années après les événements décrits?

Carnet original :

«28. - Hiniù e han hoah de Fismes. Trémen e hramb dré ouilly hag en ur arriù get sukrdi Villers Haute Rive é choman soéhet é huélet marteloded koh étal er pont. Soéhetoh onn hoah a pe dostan dehè é huélet é ma labourizion porh int. Deit int a houdé miz devéhan er blé aveit seùel hag gobér a neùé er ponteu

28. C'est pour cette raison que certains poètes ou écrivains détruisent scrupuleusement notes et brouillons. L'exemple contemporain le plus célèbre en ce domaine est celui du poète Mahmoud Darwich (1941-2008).

29. Daniel Carré précise également que l'emploi par Loeiz Herrieu d'un vocabulaire le plus restreint possible lorsqu'il s'agit de décrire la guerre et tout ce qui s'y rapporte, tient de sa volonté de ne faire aucune concession à la guerre, même lexicale. Observation de Daniel Carré le 13 décembre 2013. 
diskaret a pe oè er Germaned é tonet é Frans.

Bout e zo ag er hantér (?) anehè hag a gosté en Oriant. Un adjudant labourér porh e zo doh ou houmandein hag un ingénieur gusket èl ur Lieutenant de vaisseau. »

Kammdro an Ankoù :

«28-05-1915. - É vonet da Fismes hiziv eh on boemet-mat é welet un nebeud martoloded kozh é labourat e-tal pont Villers. Labourizion-porzh int, daet du-mañ da adsevel ar pontoù. Anavout a ran lod anezhe : a Lanester int. Emaint dre-mañ a-c'houde c'hwec'h miz bennak. »

Plus de répétitions syntaxiques ou lexicales dans la version retravaillée. Dans cette dernière, la syntaxe variée donne une dynamique, un autre rythme dû également à sa concision. Cette recherche de richesse syntaxique est commune dans les littératures européennes continentales $^{30}$. En revanche, une certaine littérature anglophone se préoccupe moins des répétitions lexicales. En retravaillant à ce niveau, Loeiz Herrieu s'inscrit clairement dans une tradition littéraire d'influence française.

Ainsi, il remplace "souéhet» [étonné] du texte original par «boemet-mat» [très étonné]. Outre que «boemet » est d'un niveau de langue plus soutenu que "souéhet», son emploi introduit une nouvelle nuance. Car le sens le plus commun de «boemet» est " émerveillé, fasciné ${ }^{31}$ », même si le bas-vannetais l'utilise couramment pour signifier la surprise ou l'étonnement ${ }^{32}$. L'emploi de ce mot introduit donc une nuance positive alors que la nature positive ou négative de la surprise n'est pas immédiatement induite dans l'emploi de « souéhet».

30. Du moins dans celles que nous sommes en mesure d'observer en langue originale, soit les littératures en langue bretonne, française, allemande, anglaise et espagnole.

31. Francis FAVEREAU, Geriadur ar brezhoneg a-vremañ - Dictionnaire du breton contemporain, Morlaix, Skol-Vreizh, 1993, p. 74.

32. A. Guillevic, P. Le Goff, Geriadurig Brehoneg-Galleg [Petit dictonnaire breton-français], Brest, Ar skol vrezoneg-Emgleo Breiz, 1986, p. 4. Caudan, où était né Loeiz Herrieu, fait partie de la zone linguistique du bas-vannetais. 
En préférant « boemet », Loeiz Herrieu opère plusieurs choix. Tout d'abord, il souligne l'aspect positif de sa surprise ${ }^{33}$ et cette indication est assez forte pour ne pas devoir la répéter. Cet emploi sert le sens et le style, car l'homme étant peu enclin aux épanchements, la suppression d'une marque d'émotion (la surprise) de même qu'il supprime également «émesk hor Bretoned " [au milieu de nos Bretons], dans lequel le pronom possessif donne une sonorité un peu trop paternaliste, lui permet de se mettre en recul. En revanche, « boemet-mat », « mat » [bien] ne renforce pas l'aspect positif, " mat» ayant ici un rôle emphatique. Ce recul est également observable dans le choix du style rapporté. Certes, le récit est à la première personne mais aucun dialogue n'est retranscrit, l'auteur est acteur mais ne se met pas pour autant directement en scène par l'intimité d'un dialogue.

À quelle information se rapporte cette surprise positive ? À la rencontre avec de vieux ouvriers de l'arsenal. Dans le carnet, ces ouvriers sont pour la moitié d'entre eux originaires de la région de Lorient. Dans Kammdro an Ankoù, Loeiz Herrieu précise qu'il en connaît quelques-uns et qu'ils sont originaires de Lanester. Pourquoi opère-t-il ce changement?

Lanester se situe sur la rive gauche du Scorff, en face de Lorient. C'est une commune créée en 1909 à partir d'une division de la commune de Caudan sur laquelle se situait le Koz Kêr, la ferme familiale des Herrieu. Loeiz Herrieu se méfiait de la ville. Par ailleurs, il était très soucieux des convenances et prétendait à un entre-soi qui ne favorisait pas les échanges avec des personnes extérieures à son milieu. En effaçant la mention de Lorient, il montre sa volonté de n'associer ni la ville ni ses habitants à une émotion positive. Préciser dans Kammdro an Ankoù que ses ouvriers viennent de Lanester, qu'il en connaît quelques-uns, maintient juste la distance convenable entre le paysan, l'homme de lettres, et ces ouvriers. La surprise positive de les rencontrer tient donc moins à l'existence d'une familiarité avec

33. Sans que cela ne remette en cause notre interprétation, Francis Favereau rappelle l'étymologie de boemet, qui se rapporte à la Bohême, aux bohémiennes jeteuses de sort qui provoquent un état d'hébétude, d'engourdissement, que peut également décrire boemet ou boémé dans sa version francisée en CentreBretagne. Précision donnée par Francis Favereau, le 13 décembre 2013. 
certains d'entre eux, que du constat de la nature de leur mission. Ces ouvriers bretons de l'arsenal de Lanester sont exilés dans les terres pour construire. Dans Kammdro an Ankoù, ils reconstruisent des ponts, et la destruction de ces ponts par les Allemands n'y est plus précisée. Les Bretons de Lanester y remontent des ponts détruits par d'autres qu'eux. Une conclusion s'impose : ces compatriotes de Loeiz Herrieu sont des constructeurs et, par défaut, les autres des destructeurs !

Par ailleurs, la suppression des détails géographiques, le passage aux abords de la sucrerie, des détails militaires, la version retravaillée ne mentionnant plus l'adjudant vêtu comme un lieutenant de vaisseau, de même que Loeiz Herrieu ne juge pas primordial de nommer les régiments d'origine ${ }^{34}$ des nouveaux venus dans Kammdro an Ankoù, démontre qu'il se situe dans le champ de l'écriture littéraire et non pas historique. Un historien aurait peut-être retravaillé ces phrases mais pour préciser les détails historiques et non pas délivrer, insister sur sa vision personnelle comme le fait Loeiz Herrieu. L'historien prétend s'en tenir à la réalité des faits. L'écrivain délivre, propose sa représentation de cette même réalité.

Cependant, la racine de ce récit étant l'aventure d'un homme dans un contexte historique extraordinaire, il n'est pas interdit de se poser la question du réalisme, de l'authenticité, si chère au critique Jean Norton-Cru ${ }^{35}$, des deux versions. Laquelle des deux est la plus vraie? La plus authentique?

La langue du carnet, tout autant que celle de Kammdro an Ankoù délivre de fait une interprétation partielle parce qu'inévitablement parcellaire d'une réalité commune. Sans compter que la langue ellemême n'est jamais parfaitement exacte, du fait du décalage de signification entre le mot et l'objet qu'il désigne. De plus, l'objet désigné s'inscrit lui-même dans le processus de modification continue qui

34. Pour information, le $88^{\text {e }}$ Régiment d'Infanterie des Territoriaux était le régiment d'origine de Loeiz Herrieu et le 144e Régiment d'Infanterie était un régiment de Bordeaux. Nous n'avons pas de renseignement sur le $218^{e}$ mentionné.

35. Auteur d'un premier ouvrage critique d'envergure sur les récits de la Grande Guerre, qui a donné lieu à de nombreuses polémiques. Jean NORTON-CrU, Témoins (Essai d'analyse et de critique des souvenirs de combattants édités en français de 1915 à 1928), Nancy, Presses Universitaires de Nancy, 1993, 727 p. 
touche chaque chose et chaque être... Le carnet brut informe différemment de Kammdro an Ankoù sur l'état d'esprit de Loeiz Herrieu et la réalité qu'il décrit, mais nous ne pouvons pas hiérarchiser la véracité du message qu'il émet. Nous ne pouvons pas affirmer que l'un est plus authentique que l'autre.

La langue des carnets n'est donc pas plus authentique, réaliste que la langue littéraire. Dans le cas du récit de guerre, la langue du premier jet est avant tout un aide-mémoire qui va permettre de déclencher des souvenirs chez l'écrivain. La transformation du texte original en texte littéraire s'opère à partir d'une modification des formes, destinée à ordonner et à délivrer le message de son auteur. Cette transformation peut s'accorder à la réalité historique mais sans que ce soit l'objectif principal du discours.

Lorsque nous observons l'extrait choisi, le texte d'origine est classiquement retravaillé au niveau syntaxique, en supprimant les répétitions, ce qui nécessite de fait un enrichissement de l'expression. Ainsi, la préposition «émesk» [parmi] présente deux fois dans le texte original ne l'est plus qu'une fois dans Kammdro an Ankoù. En revanche, le très littéraire «a-douesk» [d'entre] y fait son apparition :

Carnet original :

«E Fismes en anderù-men de 3 ér é interrér 15 sudard lahet deu zé kent é gar Fismes get ur bomb taulet d'un aerlestr hag e ziskoé bout un aerlestr a Frans. En ou mesk é oè sudarded ag er 88 hag ur serjant Durand a neùéso hanùet ér ravitaillemant léh ma um gavé a drès er peurkèh. Donet e hra hoah un aerlestr germanek de valé drest ker meit monet e hra unan ag hor ré ar é lerh ha ne déhas ket turel bomb erbet.

Guélet e hran deu di e zo koéhet bombeu arnehé. Goal auzet int, muioh memb eit get obuseu.

E tonet d'er gér é kavan hoah er labourizion porh é kampen un tachad aveit lakat ou hamakeu a skrebill aveit monet de gousket. (*) Chom e hreint aman un tér suhun benak.

E Euilly é ma arriù er 144 de gemér hor lèh. Lan é er vorh anehé. Kement-men e senefi, kredan, ne vemb ket galùet d'hobér labour en active. Arriù zo genemb territ. ag en 218 e zo lammet ag en active aveit lakaat er 88 er lod muian anehè e zo a gosté Pau. Tud didrous, um gav un tammig divroet émesk hor Bretoned e zalh de gonz ieh er vro.» 
Kammdro an Ankoù :

«E Fismes e tegouezhan just evit gwelet interamant 15 soudard lazhet er gar get ur vombezenn taolet ag un aerlestr hag a oa hañval-mat doc'h hor re. Unan bennak skrapet get ar Jermaned moarvat.

E-mesk ar geizh-se eh oa tud ag an 88vet. Unan anezhe, ur serjant, kaset du-mañ an deizioù kent hag eürus bout pellaet doc'h an talva, a zo daet amañ da glask e varv.

A pa zistroan da Beaurieux eh eus labour arall doc'h man gortoz : soudarded lamet a-douesk ar re yaouank en arbenn d'o oad a zo kaset deomp. Euskariz int, lod anezhe; tud didrouz, o-unan un tammig, hag a vourr é komz o yezh.

Kement-mañ a ra koñfort deomp : revendailh ne vezer ket soñjet d'ober genomp labour ar re yaouank. »

Quant à la numérotation précise des régiments, hormis le $88^{\mathrm{e}}$, elle a disparu et plus généralement, les détails précis sur la vie militaire sont mis en arrière-plan. L'information sur le bombardement de la gare, sur le vol de l'avion est maintenue dans Kammdro an Ankoù, parce qu'elle explique l'enterrement. Mais la différence des dégâts provoqués par la bombe ou l'obus, la description de la suspension du hamac par les ouvriers de l'arsenal sont supprimées. Cela confirme une nouvelle fois que Loeiz Herrieu se veut écrivain, témoin et rapporteur d'événements historiques à son niveau et non pas historien, puisqu'il privilégie l'esthétique propre à la littérature qui peut voir disparaître un détail historique s'il ne sert pas le discours littéraire.

Évidemment, le processus provoquant cette sélection interroge et nous ramène à la période de rédaction de Kammdro an Ankoù. En 1933, les Français qui s'intéressent au sujet ont déjà vu la publication d'un nombre incroyable de témoignages, de romans, sur la Grande Guerre. À cause de cela, la profusion de détails sur la vie militaire peut rebuter un lecteur déjà saturé par l'inflation des récits de guerre. Loeiz Herrieu n'a donc pas besoin de livrer ces détails intéressants, mais seulement du point de vue historique. D'autant plus qu'ils risquent d'alourdir le récit littéraire s'ils ne servent pas à éclairer tel ou tel événement, en l'occurence un enterrement dans le passage cité.

Dans ce passage cependant, cette préoccupation de décrire l'homme avant le militaire est curieusement contredite par la dis- 
parition de l'identité du sergent Durand, nommé dans le carnet et devenu anonyme dans Kammdro an Ankoù, alors que Loeiz Herrieu y cite par ailleurs abondemment ses amis et de nombreux compagnons de combat. Cette éviction est du même ordre que l'évocation des ouvriers de l'arsenal de Lanester. Dans Kammdro an Ankoù, il n'est plus question de la moitié d'entre eux. Loeiz Herrieu en connaît quelques-uns et ceux-ci sont de Lanester. Nous savons que dans ce passage il veut mettre en avant le travail constructif de ces Bretons. Le $88^{\mathrm{e}}$ régiment auquel appartient Loeiz Herrieu, duquel est issu le sergent Durand, est principalement composé de Bretons. En réduisant le sergent Durand à un sergent anonyme provenant du 88, Loeiz Herrieu laisse désormais penser que ce sergent était breton. Or, la mention d'un nom aussi français que Durand aurait pu suggérer un éloge du courage d'un Français par l'auteur. Loeiz Herrieu n'a rien contre les Français, à condition qu'ils restent à leur place : c'està-dire en France, qu'il distingue de la Bretagne. Loeiz Herrieu est partisan de l'entre-soi. Les Bretons doivent vivre entre eux en Bretagne et décider du sort de la Bretagne. Cela s'entend aussi pour les Parisiens à Paris, les Basques dans le Pays basque... Cette volonté d'entre-soi s'applique également sur le terrain social. De ce fait, il a exclu une référence trop explicite à des citadins dont il se méfie, la ville étant le creuset des mélanges culutrels et sociaux qui affaiblissent la société traditionnelle aux traditions séculaires à préserver. Des citadins lorientais en l'occurrence, qu'il serait regrettable de présenter en reconstructeurs, de la même manière qu'il ne serait pas convenable de faire une telle publicité aux Français ${ }^{36}$.

La mention disparue de l'origine paloise des soldats qui rejoignent le $88^{\mathrm{e}}$, devenus uniquement des Basques, qui parlent leur langue, est effectuée exactement dans le même esprit. Elle tient à la volonté de distinguer les Basques des autres Français, en montrant ici leur particularité linguistique. Par ailleurs, nourrissant de solides préjugés contre les gens du Sud de la France (Basques mis à part), Loeiz Herrieu efface la confusion pouvant naître à la lecture de la mention de la ville d'origine du régiment, qui pourrait laisser croire

36. Bien que le pauvre sergent Durand fût peut-être un Breton. La symbolique associée à son nom passe avant l'homme. 
à une sorte de publicité pour des soldats originaires du Sud, tout comme il l'avait fait avec le nom du soldat Durand.

Enfin, dans ce passage de Kammdro an Ankoù, Loeiz Herrieu rappelle la conjonction entre son esprit nationaliste et le catholicisme tel qu'il l'entend, quand il insiste sur son arrivée in extremis à l'enterrement. «E Fismes e tegouezhan just evit gwelet interamant (...) » [« Nous arrivons à Fismes juste pour voir l'enterrement (...)»]. Dans le carnet, il mentionne cet enterrement sans s'y impliquer, en le présentant d'un impersonnel "é interrér » [on enterre]. Cette impersonnalité disparaît dans Kammdro an Ankoù, et Loeiz Herrieu insiste au moyen de l'adverbe «just» [juste], sur l'importance qu'il accorde à cette cérémonie. Il rappelle ici son attachement au rite chrétien d'accompagnement des morts. Le chiffrage dans les deux textes du nombre des morts, la suppression d'un patronyme à consonance trop française pour laisser penser que tous ces soldats étaient bretons sont destinés à rappeler la hauteur du sacrifice des Bretons «venus chercher ici leur mort». En plus du rappel, par la mention des Basques, que les autres sacrifiés étaient originaires d'autres « nations » que la France.

Les préoccupations de Loeiz Herrieu en 1915 ne sont pas radicalement différentes de celles qu'il décrit en 1933. Mais certaines ont changé d'échelle et l'environnement qu'il a retrouvé en Bretagne est radicalement différent du front. Et puis, l'expérience de cette guerre l'amène à mettre en avant différentes revendications dans un contexte de radicalisation politique général en Europe.

Dans le cas de Loeiz Herrieu, cette radicalisation est parfaitement décrite par Daniel Carré :

« Le discours de Loeiz se radicalise dans Dihunamb à partir de la fin des années 1920 : le combat pour la survie de la langue est vital si l'on veut préserver l'identité bretonne ; la Bretagne a le droit de s'opposer à la France qui étouffe sa personnalité et refuse de reconnaître le sacrifice que la Bretagne a consenti pour sa défense ; si la France continue à lui nier ce droit, les Bretons doivent se préparer à le prendre de force lorsque l'opportunité se présentera $^{37}(\ldots)$. »

37. Daniel CARRÉ, Loeiz Herrieu, un paysan breton dans la grande guerre..., op. cit. 
La façon dont Loeiz Herrieu a retravaillé ses carnets, rédigés dans une langue spontanée, pour les transformer en un récit dans une langue littéraire, participe d'une certaine manière à cette radicalisation. Car au travers de certains choix d'effacer, de modifier la description des événements et la manière de présenter, de nommer des hommes ou des groupes d'hommes, nous observons un engagement politique plus prononcé qu'au moment où les carnets ont été écrits.

Cette transformation nous éclaire surtout sur la permanence de la vérité de cet homme, plongé dans un environnement différent à deux périodes de sa vie. Et si les choix qu'il opère dans sa description partent d'une réalité commune à tous les combattants, c'est avant tout la vérité singulière d'un individu rapportée avec sa voix singulière qui apparaît dans Kammdro an Ankoù, comme dans toute œuvre littéraire.

Pour finir, il convient de mentionner et de remercier les chercheurs qui nous ont permis de corriger, de nuancer, d'approfondir certains points présentés, en un mot d'enrichir cet article, grâce à leurs observations lors de notre communication du vendredi 13 décembre 2013 à l'Université de Bretagne Occidentale, dans le cadre du séminaire La Bretagne Linguistique. Nous adressons donc nos remerciements aux chercheurs et professeurs suivants : Daniel Carré, Erwan Le Pipec, Mélanie Jouitteau, Jo Rio, Francis Favereau et Ronan Calvez. 